\title{
Tuning nanoparticle size for enhanced functionality in perovskite thin films deposited by metal organic deposition
}

\begin{abstract}
Masashi Miura ${ }^{1,2}$, Boris Maiorov ${ }^{1}$, Michio Sato $^{2}$, Motoki Kanai ${ }^{2}$, Takeharu Kato ${ }^{3}$, Tomohiro Kato ${ }^{3}$, Teruo Izumi ${ }^{4}$, Satoshi Awaji ${ }^{5}$, Paolo Mele ${ }^{6}$, Masaru Kiuchi ${ }^{7}$ and Teruo Matsushita ${ }^{7}$

Because of pressing global environmental challenges, focus has been placed on materials for efficient energy use, and this has triggered the search for nanostructural modification methods to improve performance. Achieving a high density of tunable-sized second-phase nanoparticles while ensuring the matrix remains intact is a long-sought goal. In this paper, we present an effective, scalable method to achieve this goal using metal organic deposition in a perovskite system $\mathrm{REBa}_{2} \mathrm{Cu}_{3} \mathrm{O}_{7}(\mathrm{rare}$ earth (RE)) that enhances the superconducting properties to surpass that of previous achievements. We present two industrially compatible routes to tune the nanoparticle size by controlling diffusion during the nanoparticle formation stage by selecting the second-phase material and modulating the precursor composition spatially. Combining these routes leads to an extremely high density $\left(8 \times 10^{22} \mathrm{~m}^{-3}\right)$ of small nanoparticles $(7 \mathrm{~nm})$ that increase critical currents and reduce detrimental effects of thermal fluctuations at all magnetic field strengths and temperatures. This method can be directly applied to other perovskite materials where nanoparticle addition is beneficial.
\end{abstract}

NPG Asia Materials (2017) 9, e447; doi:10.1038/am.2017.197; published online 17 November 2017

\section{INTRODUCTION}

The world's energy demands require more efficient methods of producing, storing, transmitting and using clean energy, such as electricity. Superconductors can help address all of these areas with more efficient generators/wind turbine generators, magnetic energy storage devices, power transmission lines, electric motors and so on. One key for these applications is to develop materials such as superconductor, photoelectric and thermoelectric materials that enable alternative energy 'ecosystems'. For all of these material types, it is necessary to find industrially compatible ways to fabricate high-performance material. The method of nanocomposite inclusion in different perovskite materials is highly effective. Superconductive/ thermoelectric material performance can be improved by adding nanoparticles (NPs) that disrupt the order parameter/phonon density while leaving the matrix and electronic structure of the superconductor/thermoelectric intact. ${ }^{1-4}$ In the case of thermoelectrics, the reduction of lattice thermal conductivity through phonon scattering by size-controlled NPs has been predicted in the seminal paper by Hicks and Dresselhaus. ${ }^{5}$ This approach has already been demonstrated to be effective in metallic materials, such as $\mathrm{Bi}_{2} \mathrm{Te}_{3} / \mathrm{Sb}_{2} \mathrm{Te}_{3}$ multilayers ${ }^{6}$ and $\mathrm{PbTe}$ bulk material ${ }^{1}$ with natural NP additions with a significant enhancement of the figure of merit (ZT) that has reached up to $\sim 2$ at
$800 \mathrm{~K}$. In comparison with metallic materials, oxides offer the advantages of reduced cost, nontoxicity and higher stability at high temperatures. Impressive performance using a nanoengineering approach has been obtained in $\mathrm{SrTiO}_{3}$ multilayers with added $\mathrm{Nb}$ nanoparticles. ${ }^{7}$ Thin films oxides, such as hexagonal $\mathrm{Al}-\mathrm{ZnO},{ }^{8}$ perovskite $\mathrm{RE}_{2-x} \mathrm{Ce}_{x} \mathrm{CuO}_{4}(\mathrm{RE} 214)^{2,9}$ and $\mathrm{RE}$-doped $\mathrm{Sr}_{3} \mathrm{Ti}_{2} \mathrm{O}_{7},{ }^{10}$ have already demonstrated excellent thermoelectric properties even without the addition of NPs. Other materials, such as transparent delafossite $\mathrm{CuAlO}_{2},{ }^{11}$ have the potential to be used as thermoelectrics. The paradigm of insertion and control of artificial NPs in high- $T_{\mathrm{c}}$ superconductors can be easily transferred to all of these other oxides to prepare efficient, environmentally friendly thermoelectric harvesters. It is also crucial to achieve this in a scalable, high-throughput (that is, economical) way. Metal organic deposition (MOD) fulfills all of these requirements; thus, finding ways to tailor the size and density of NPs while ensuring that the underlying matrix remains as pristine as possible is a goal that has been pursued for decades.

After the seminal works demonstrated that second-phase additions $^{4,12}$ to RE-Ba-Cu-O (rare earth (RE)) (REBCO) thin films grown using pulsed laser deposition (PLD) significantly improved the current carrying performance in an applied magnetic field, ${ }^{3,13-15}$ it was shown that the morphology of the inclusions depends on the synthesis

\footnotetext{
${ }^{1}$ Materials Physics and Applications Division, Los Alamos National Laboratory, Los Alamos, NM, USA; ${ }^{2}$ Graduate School of Science and Technology, Seikei University, Tokyo, Japan; ${ }^{3}$ Nanostructures Research Laboratory, Japan Fine Ceramics Center, Nagoya, Japan; ${ }^{4}$ National Institute of Advanced Industrial Science and Technology, Ibaraki, Japan; ${ }^{5}$ High Field Laboratory for Superconducting Materials, IMR, Tohoku University, Miyagi, Japan; ${ }^{6}$ Muroran Institute of Technology, Hokkaido, Japan and ${ }^{7}$ Kyushu Institute of Technology, Fukuoka, Japan

Correspondence: Professor M Miura, Graduate School of Science and Technology, Seikei University, 3-3-1 Kichijoji-kitamachi, Musashino-shi, Tokyo 180-8633, Japan. E-mail: masashi-m@st.seikei.ac.jp

Received 31 August 2017; revised 13 September 2017; accepted 17 September 2017
} 
a

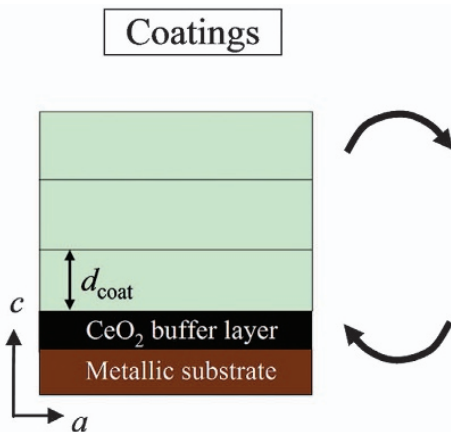

\section{Calcinations}

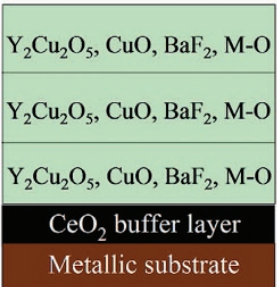

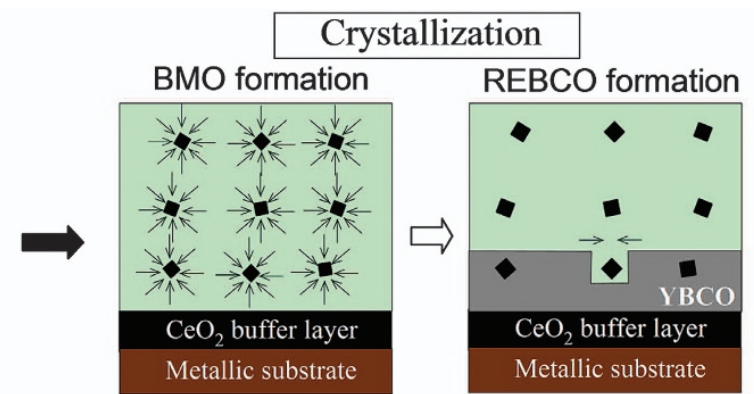

b
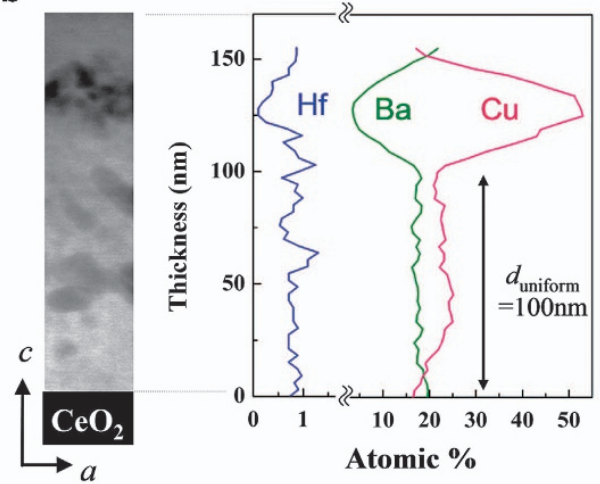

Atomic \%

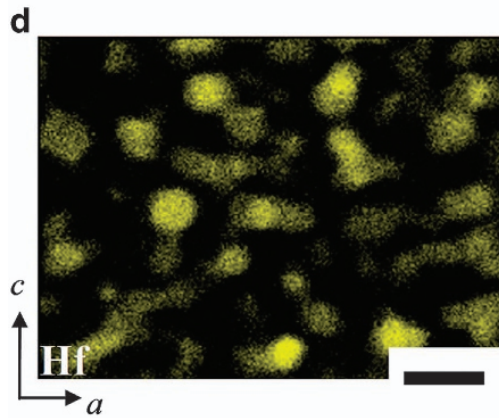

e

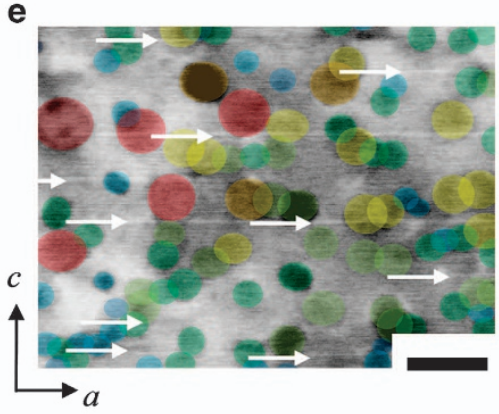

C

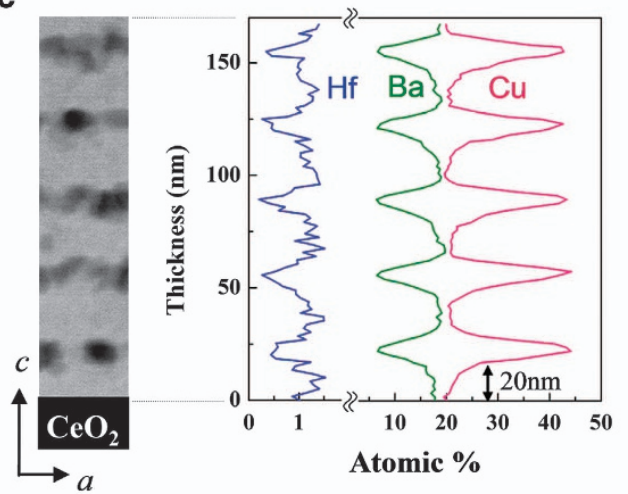

f

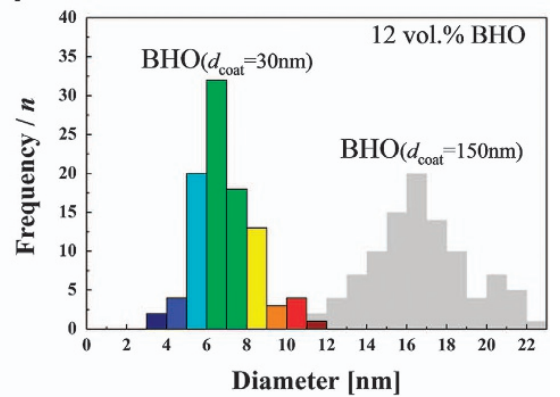

Figure 1 Metal organic deposition (MOD) process and cross-sectional microstructure of calcined and crystallized (Y,Gd)BCO+12BHO films. (a) Schematic of the MOD process for fabrication of $\mathrm{BaMO}_{3}$ (BMO) nanoparticle (NP)-doped (Y,Gd)BCO films. (1) Coating a chemical solution; (2) calcination to obtain a precursor containing several amorphous phases; and (3) crystallization of the superconducting phase. Cross-sectional high-angle annular dark-field (HAADF) images and compositional line analyses of $\mathrm{Hf}, \mathrm{Ba}$ and $\mathrm{Cu}$ for the calcined films in $+12 \mathrm{BHO}$ film with (b) $d_{\text {coat }}=150$ and (c) $30 \mathrm{~nm}$, respectively. The horizontal axis indicates the distance from the $\mathrm{CeO}_{2}$ buffer layer. (d) Elemental maps of $\mathrm{Hf}$ and (e) cross-sectional scanning transmission electron microscopy $(\mathrm{STEM})$ image for the $+12 \mathrm{BHO}\left(d_{\text {coat }}=30 \mathrm{~nm}\right)$ film. The horizontal bar scale indicates $20 \mathrm{~nm}$ for both $(\mathbf{d}, \mathbf{e})$. In (e), BaHfO $3(\mathrm{BHO})$ nanoparticles are color-coded by size. The horizontal arrows in (e) indicate stacking faults observed around the nanoparticles. (f) Histograms showing the distribution of diameters of BHO NPs for $+12 \mathrm{BHO}$ films with $d_{\text {coat }}=150$ and $30 \mathrm{~nm}$. The colors correspond to those in (e).

method (that is, in situ vs ex situ). ${ }^{3,16}$ Moreover, recent work has shown that large quantities $(\geqslant 10 \%)$ of second-phase additions are required to obtain the desired level of performance in superconductors. ${ }^{17-19}$ Until now, such a large amount of second phase resulted in current blocking and the loss of crystallinity. ${ }^{19,20}$

For REBCO superconductors, MOD has been shown to be amenable for growing extremely high performance materials ${ }^{21-26}$ with high throughput; ${ }^{27-29}$ however, the tailoring of NP inclusions is extremely difficult. ${ }^{30}$ Unlike PLD, the formation of NPs during MOD occurs before matrix formation; therefore, the NP size is not determined by crystalline strain but by diffusion, leaving little room for tailoring particle size compared with that of in situ processes, where growth dynamics affect the nanoparticle/nanorod morphology. ${ }^{31,32}$ Because the REBCO crystallization process in MOD is liquid mediated, the nanoparticle-matrix interphase is incoherent; thus, the matrix crystalline quality is less affected (than for PLD), allowing for higher NP densities.

Here, we report a new strategy to tailor an ultra-high density of tunable-size, incoherent-interfaced $\mathrm{BaHfO}_{3}(\mathrm{BHO}) \mathrm{NPs}$ that result in enhanced properties for perovskite-composite cuprate superconductor $\left(\mathrm{Y}_{0.77} \mathrm{Gd}_{0.23}\right) \mathrm{Ba}_{2} \mathrm{Cu}_{3} \mathrm{O}_{y}((\mathrm{Y}, \mathrm{Gd}) \mathrm{BCO})$ films. This strategy limits the growth of second-phase NPs by reducing diffusion during formation and produces an Hf- and Ba-poor layer by using multiple MOD coating/calcination steps. The high density of BHO NPs results in virtually no degradation of the matrix crystallinity as evidenced by the further improved superconducting properties. Critical current density $J_{\mathrm{c}}(\theta, H, T)$ measurements demonstrate that the NPs are highly effective as pinning centers by decreasing vortex motion and depinning 

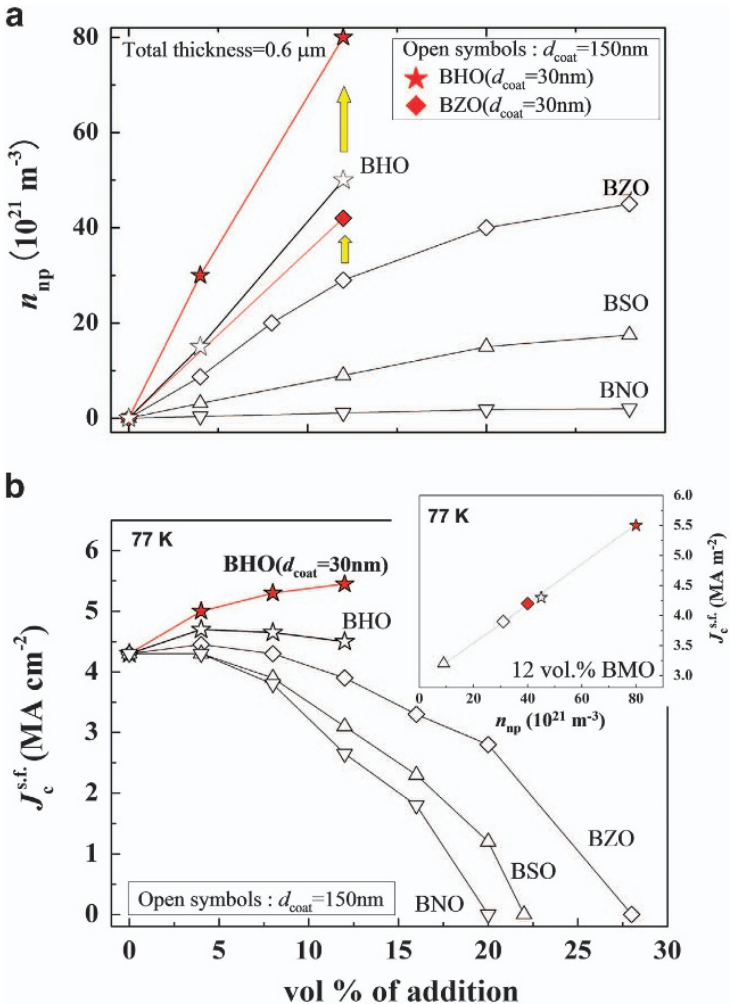

Figure 2 Nanoparticle (NP) density and self-field $J_{c}$ as a function of vol.\% of addition for $(\mathrm{Y}, \mathrm{Gd}) \mathrm{BCO}+\mathrm{BMO}$ films. (a) $\mathrm{BaMO}_{3}$ (BMO) NP density as a function of volume percent of addition for $(\mathrm{Y}, \mathrm{Gd}) \mathrm{BCO}+\mathrm{BMO}$ films obtained from several transmission electron microscopy (TEM) images. Open and solid symbols represent data of films with $d_{\text {coat }}=150$ and $30 \mathrm{~nm}$, respectively. (b) $J_{c}$ s.f. at $77 \mathrm{~K}$ as a function of volume percent of addition for $(\mathrm{Y}, \mathrm{Gd}) \mathrm{BCO}$ +BMO films. Inset: J J.f. at $77 \mathrm{~K}$ versus NP density for films with 12 vol.\% of different dopants.

fluctuation effects for all magnetic field strengths and orientations. Furthermore, we present a simple pinning model that predicts the superconductor performance with NPs for any temperature and magnetic field using only a small set of fixed parameters. Our synthesis method can be directly applied to other perovskitecomposite materials to improve their functionality.

\section{MATERIALS AND METHODS}

\section{$\left(\mathrm{Y}_{0.77} \mathrm{Gd}_{0.23}\right) \mathrm{Ba}_{2} \mathrm{Cu}_{3} \mathrm{O}_{y}$ films with a high density of NPs}

HTS wires based on epitaxially grown REBCO nanocomposite films of standard (Y,Gd)BCO and BMO NP-doped films were grown from metal organic solutions that included Y-, Gd-, Ba-trifluoroacetates, and $\mathrm{Cu}$-naphthenate with a cation ratio of $0.77: 0.23: 1.5: 3$ on buffered tapes of $\mathrm{CeO}_{2}$ (grain-boundary angles, $\left.\Delta \phi=3.0^{\circ}\right) / \mathrm{Y}_{2} \mathrm{O}_{3} / \mathrm{LaMnO}_{3} /$ ion-beam-assisted deposition- $\mathrm{MgO} / \mathrm{Gd}_{2} \mathrm{Zr}_{2} \mathrm{O}_{7} /$ Hastelloy C276 (Haynes International Inc., Kokomo, IN, USA). We added M-naphthenate $(\mathrm{M}=\mathrm{Zr}, \mathrm{Nb}, \mathrm{Sn}$ or $\mathrm{Hf})$ into the $(\mathrm{Y}, \mathrm{Gd}) \mathrm{BCO}$ solutions; the volume percent of $\mathrm{BaZrO}_{3}$ (BZO) was $4-28 \%$, and the concentration of the starting solution was $1.2 \mathrm{~mol}^{-1}$ for $d_{\text {coat }}=150 \mathrm{~nm}$ films. For $d_{\text {coat }}=30 \mathrm{~nm}$ films, the concentration of the starting solution was $0.45 \mathrm{moll}^{-1}$. The coated film was thermally decomposed to an amorphous phase precursor by heating up to $500{ }^{\circ} \mathrm{C}$ at a heating rate of $5^{\circ} \mathrm{C} \mathrm{min}^{-1}$ in a humid oxygen atmosphere $\left(P\left(\mathrm{H}_{2} \mathrm{O}\right)=3.2 \mathrm{kPa}\right.$ with an inlet temperature of the humid oxygen gas of $300^{\circ} \mathrm{C}$ ). The number of coating/calcination cycles to obtain the final film thickness of $0.6 \mu \mathrm{m}$ was 4 and 20 for the $d_{\text {coat }}=150 \mathrm{~nm}$ and $d_{\text {coat }}=30 \mathrm{~nm}$ films, respectively. The calcined film was heated up to $740{ }^{\circ} \mathrm{C}$ at $20^{\circ} \mathrm{C} \mathrm{min}^{-1}$ and held for $80 \mathrm{~min}$ in a humid oxygen gas environment for crystallization and conversion to the REBCO phase material. The total thickness of the superconductive layer for all samples was $0.6 \mu \mathrm{m}$, confirmed by cross-sectional transmission electron microscopy (TEM). Additional details of the calcinations and conversion steps have been published elsewhere. $^{30}$

\section{Transport properties in magnetic fields}

Films were patterned using photolithography and ion milled into bridges with widths of $\sim 50 \mu \mathrm{m}$. The crystalline quality was examined by X-ray diffraction. The temperature dependence of the resistivity $(\rho)$ was measured by a four-probe method in the temperature range of 10-300 K using a Physical Property Measurement System (PPMS, Quantum Design, Inc., San Diego, CA, USA) with a superconducting magnet generating a field $\mathbf{H}$ of up to $9 \mathrm{~T}$. In the PPMS, a rotational stage was used to rotate the samples with respect to $\mathbf{H}$. The critical current was determined using a $1 \mu \mathrm{V} \mathrm{cm}{ }^{-1}$ criterion.

\section{Microstructure and chemical composition analysis}

The samples were thinned by $\mathrm{Ga}$ ions at an accelerating voltage ranging from 40 to $2 \mathrm{kV}$ in a focused ion beam scanning electron microscopy dual-beam system (NB5000, Hitachi High-Technologies Co., Tokyo, Japan) equipped with a microsampling system to prepare cross-sectional and plan-view TEM specimens. The focused ion beam damaged layers formed on the TEM specimens were removed using an $\mathrm{Ar}$ ion beam at an accelerating voltage of $1 \mathrm{kV}$ in a PIPS39 Model 691 (Gatan, Inc. Pleasanton, CA, USA). The microstructure and local chemical composition of the specimens were investigated using a EM-002BF and a JEM-2100F (Topcon Co., Tokyo, Japan) with an aberration-corrected scanning system at an accelerating voltage of $200 \mathrm{kV}$. Energy-dispersive X-ray spectroscopy elemental maps were acquired by a Noran System Seven (Hitachi High-Technologies Co.) with twin energydispersive X-ray spectroscopy detectors in a microscope (Topcon Co., Tokyo, Japan).

\section{RESULTS AND DISCUSSION}

\section{Routes to tune the nanoparticle size in MOD films}

Superconductor films (Y,Gd)BCO are grown on metallic substrates using MOD. As described in Figure 1a, the standard MOD process consists of coatings, calcinations and crystallization. Early in the temperature ramp of the crystallization, $\mathrm{BaMO}_{3}(\mathrm{BMO}, \mathrm{M}=\mathrm{Zr}, \mathrm{Nb}$ or $\mathrm{Sn}$ ) NPs are formed before the crystalline growth of the matrix, resulting in randomly distributed and oriented NPs. ${ }^{21,33}$ Previously, we demonstrated that by changing $\mathrm{M}$ in $\mathrm{BMO}$, we could control the NP size from 22 to $83 \mathrm{~nm}^{30}$ but did not determine the mechanism behind the different NP sizes.

In this paper, we present a new process that limits NP growth by creating Ba- and M-poor regions leveraged by even smaller NPs using $\mathrm{M}=$ Hf. These regions appear at the interface between coatings that thus reduces the precursor thickness $\left(d_{\text {coat }}\right)$ and produces smaller NPs. The BHO NP-doped films were grown from 4,8 and 12 vol.\% BHO-doped MOD solutions. Figures $1 b$ and $c$ show the crosssectional high-angle annular dark-field images and the compositional line analysis results of the calcined films quenched after multiple coating/calcination steps with different $d_{\text {coat }}$ values for $12 \mathrm{vol} . \%$ BHO additions. To obtain a film total thickness of $0.6 \mu \mathrm{m}$, the number of coating/calcination cycles required was 4 and 20 for the $d_{\text {coat }}=150 \mathrm{~nm}$ and $d_{\text {coat }}=30 \mathrm{~nm}$ films, respectively. The thickness profiles of the layer composition are uniform up to a height $\left(d_{\text {uniform, }}\right.$ proportional to $\left.d_{\text {coat }}\right)$ above which the layer is $\mathrm{Cu}$ rich and $\mathrm{Ba}$ and $\mathrm{Hf}$ poor. A cross-sectional Hf elemental map and a TEM image for a 12 vol. $\%$ BHO-doped (Y,Gd)BCO with $d_{\text {coat }}=30 \mathrm{~nm} \quad(+12 \mathrm{BHO}$ $\left.\left(d_{\text {coat }}=30 \mathrm{~nm}\right)\right)$ is shown in Figures $1 \mathrm{~d}$ and e, respectively, showing the presence of uniformly dispersed NPs. Figure 1f shows the particlesize distribution extracted from several planar (not shown) and crosssectional scanning TEM images. Films with $d_{\text {coat }}=150 \mathrm{~nm}$ have a broad NP distribution with an average size of $17 \pm 5 \mathrm{~nm}$. However, for $d_{\text {coat }}=30 \mathrm{~nm}$, the distribution is much sharper with an average 
a

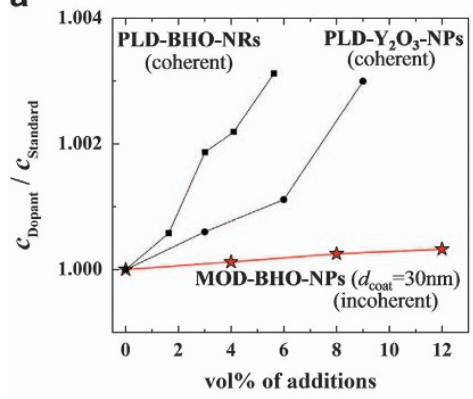

b

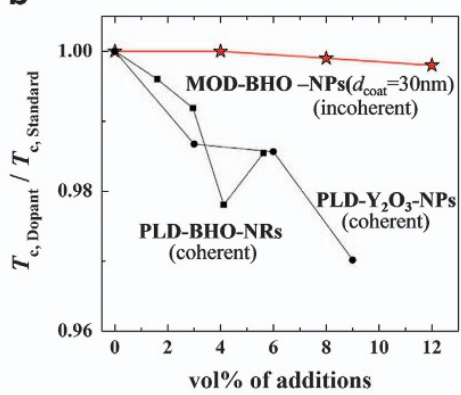

C

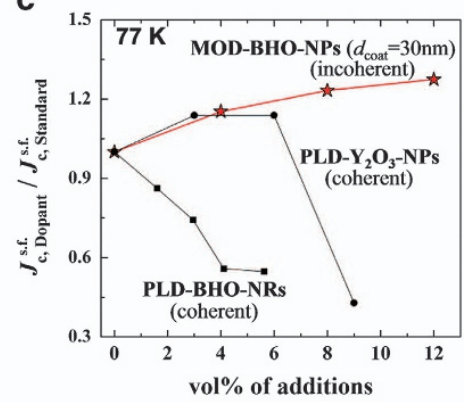

d

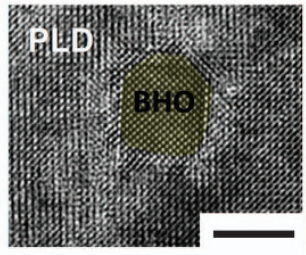

e

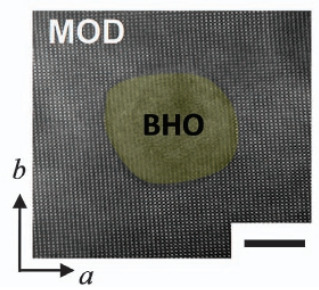

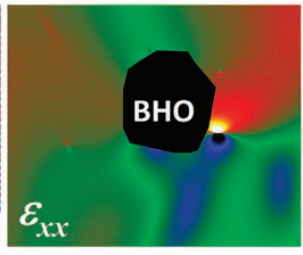

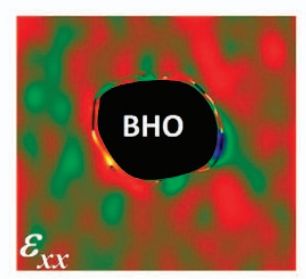

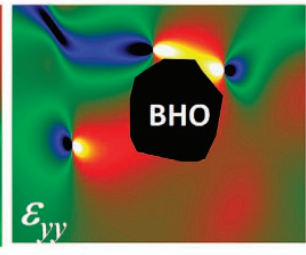

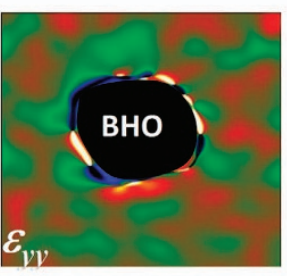

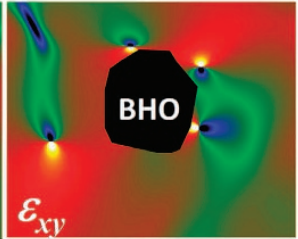

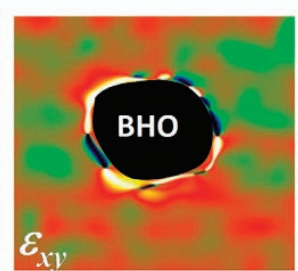

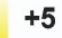

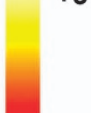

@

Figure 3 Structural and superconducting properties as a function of vol.\% of additions and strain maps for pulsed laser deposition (PLD) and metal organic deposition (MOD) films. (a-c) Normalized $c$-axis length, $T_{\mathrm{c}}$ and $J_{\mathrm{c}}$ s.f. at $77 \mathrm{~K}$ as a function of vol.\% of additions, respectively. (d,e) High-resolution plan-view images, where $\varepsilon_{\mathrm{xx}}, \varepsilon_{\mathrm{yy}}$ and $\varepsilon_{\mathrm{xy}}$ maps were determined by geometrical phase analysis for $\mathrm{BaHfO}_{3}(\mathrm{BHO})$ nanorod (NR)-doped PLD-GdBCO and $+12 \mathrm{BHO}$ $\left(d_{\text {coat }}=30 \mathrm{~nm}\right)$ films, respectively. The horizontal bar scale indicates $5 \mathrm{~nm}$ for both $(\mathbf{d}, \mathbf{e})$.

NP size of $7 \pm 3 \mathrm{~nm}$, an average NP spacing of $L_{\mathrm{NP}} \sim 20 \mathrm{~nm}$ and a density of $n_{\mathrm{np}} \sim 8 \times 10^{22} \mathrm{~m}^{-3}$. The Ba- and Hf-poor regions act as barriers to NP coarsening because elements diffuse and NPs nucleate and grow in the beginning of crystallization; a thicker $d_{\text {uniform }}$ allows for additional coarsening of the NPs (essentially semi-infinite diffusion), yielding larger particles and a wider size distribution compared with less diffusion, smaller size and a narrower distribution for smaller $d_{\text {uniform values. }}$

In Figure 2a, we show the dependence of $n_{\mathrm{np}}$ on the vol.\% and dopant material in (Y,Gd)BCO films. Here, $n_{\mathrm{np}}$ varies systematically with $\mathrm{M}$ and increases for smaller $d_{\text {coat }}$ values. The $n_{\mathrm{np}}$ of $+12 \mathrm{BHO}$ films increased by a factor of 2 between $d_{\text {coat }}=150$ and $30 \mathrm{~nm}$. The increase of $n_{\text {np }}$ between $d_{\text {coat }}=150$ and $30 \mathrm{~nm}$ for $+12 \mathrm{BZO}$ films is a factor 1.5. Similar behavior between $\mathrm{BHO}$ and $\mathrm{BZO}$ additions indicates that the technique is general and can be applied to control the BMO NP size in perovskite-composite materials. The NP size also depends on $\mathrm{M}(=\mathrm{Nb}, \mathrm{Sn}, \mathrm{Zr}$ and $\mathrm{Hf})$ that ranges from $83 \mathrm{~nm}$ for $\mathrm{BaNbO}_{3}(\mathrm{BNO})^{30}$ down to $17 \mathrm{~nm}$ for $\mathrm{BaHfO}_{3}$ in 4 vol. $\%$ doped (Y,Gd)BCO with $d_{\text {coat }}=150 \mathrm{~nm}$. Although a similar dependence on size of BMO nanorods (NRs) is observed in PLD films ${ }^{34}$ the size-determination mechanisms are not necessarily the same because the growth mechanisms are clearly different. The BMO NRs in PLD grow with the REBCO phase in the same growing interface, and therefore the growth of each phase is strongly influenced by the other phase ${ }^{35}$ (coherent interfaces). On the other hand, BMO NPs in MOD freely nucleate and grow in the precursors before the growth of the REBCO phase (incoherent interfaces). Nevertheless, diffusion plays a dominant role for NP formation. From X-ray diffraction patterns of films quenched at different temperatures during crystallization, we observed the $\mathrm{BMO}$ formation temperature $T_{\mathrm{BMO}}$ to be $\sim 740, \sim 720, \sim 700$ and $\sim 630^{\circ} \mathrm{C}$ for $\mathrm{BNO}, \mathrm{BaSnO}_{3}, \mathrm{BZO}$ and $\mathrm{BHO}$, respectively, indicating a clear correlation between a lower $T_{\mathrm{BMO}}$ and a smaller NP size. Our findings indicate that the formation temperature, which dictates diffusion, provides another route to tune the NP size.

Figure $2 \mathrm{~b}$ shows the self-field $J_{\mathcal{c}}\left(J_{\mathrm{c}}^{\text {s.f. }}\right)$ as a function of vol.\% of addition. In particular, no decrease in $J_{\mathrm{c}}^{\text {s.f. }}$ with $\mathrm{BHO}$ additions $\left(d_{\text {coat }}=30 \mathrm{~nm}\right)$ is found. The inset in Figure $2 \mathrm{~b}$ shows $J_{c}^{\text {s.f. }}$ initially increasing linearly with $n_{\mathrm{np}}$, consistent with single vortex strong pinning ${ }^{36,37}$ by NPs. The $J_{\mathrm{c}}^{\text {s.f. }}$ decrease for the $\mathrm{BNO}, \mathrm{BaSnO}_{3}$ and BZO films ( $>10$ vol.\%), which is related to the coarsening and interconnection of NPs, is also evidenced by the sublinearity of $n_{\mathrm{np}}$-vol.\%. These results highlight the importance of the choice of dopant material to both tune the density and control size to obtain the desired performance.

\section{Incoherent fine NP in MOD films}

Part of the successful addition of NPs in MOD can be found in Figure 3 by comparing the structural and superconducting properties for inclusions with coherent and incoherent interfaces, that is, coherent NRs in films grown using a low-temperature growth technique via PLD (PLD-BHO-NRs), ${ }^{20}$ coherent nanoparticles $\left(\mathrm{PLD}-\mathrm{Y}_{2} \mathrm{O}_{3}-\mathrm{NPs}\right)^{38}$ and incoherent nanoparticles (MOD-BHO-NPs $\left.\left(d_{\text {coat }}=30 \mathrm{~nm}\right)\right)$. For both PLD films, the $c$-axis length of the superconducting matrix lattice expands markedly with increasing vol.\% but, in contrast, remains flat for MOD-BHO-NPs. Similarly, a negative 


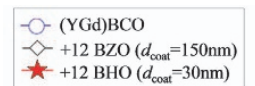

a

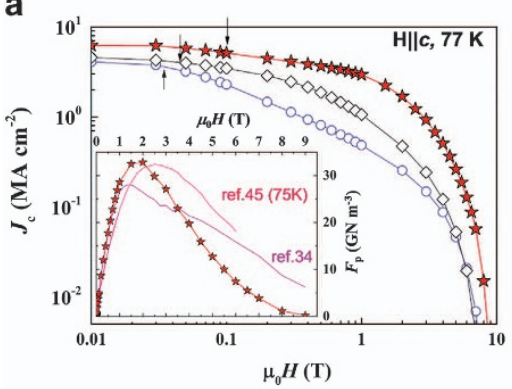

d
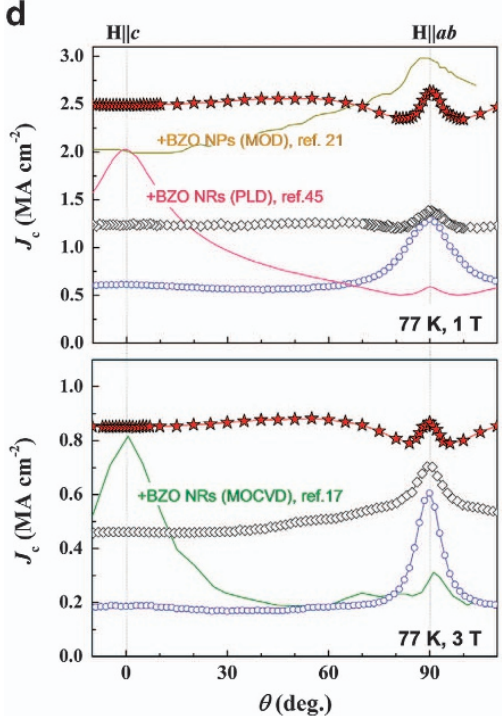

b

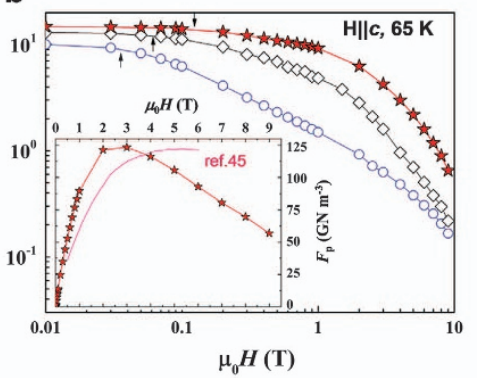

e
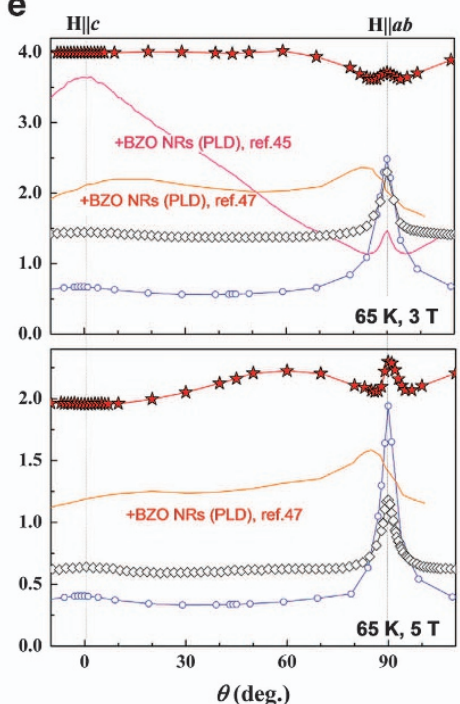

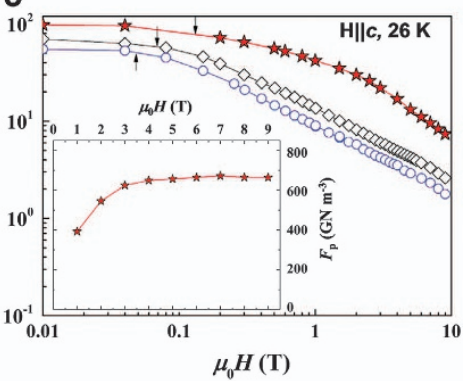

f
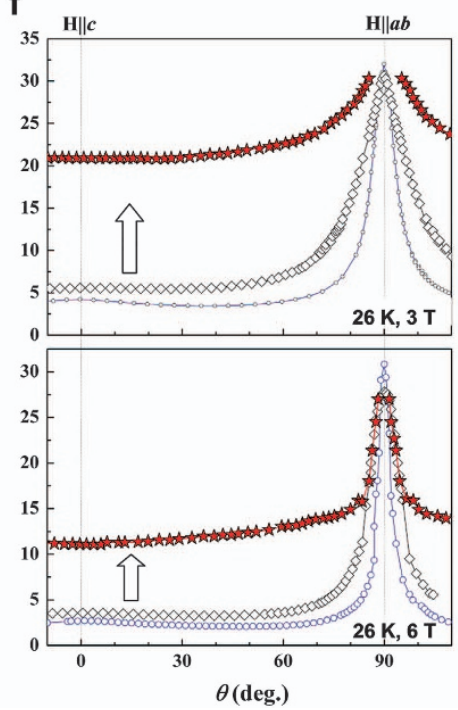

Figure 4 Critical current density as a function of magnetic field amplitude and angle. $(\mathbf{a}-\mathbf{c}) J_{\mathrm{c}}(\mathbf{H} \| \mathbf{c})$ for the $(\mathrm{YGd}) \mathrm{BCO},+12 \mathrm{BZO}\left(d_{\mathrm{coat}}=150 \mathrm{~nm}\right)$ and $+12 \mathrm{BHO}$ $\left(d_{\text {coat }}=30 \mathrm{~nm}\right)$ films at 77,65 and $26 \mathrm{~K}$, respectively. Inset: $F_{\mathrm{p}}-\mu_{0} H$ curves of the $+12 \mathrm{BHO}\left(d_{\text {coat }}=30 \mathrm{~nm}\right)$ film at each temperature. For comparison, the data for the $\mathrm{Ba}_{2} \mathrm{YNbO}_{6}$ nanorod (NR)-doped pulsed laser deposition (PLD) films ${ }^{45}$ at 65 and $75 \mathrm{~K}$ are included. (d-f) $J_{\mathrm{c}}$ as a function of angle for the (YGd) $\mathrm{BCO},+12 \mathrm{BZO}\left(d_{\text {coat }}=150 \mathrm{~nm}\right)$ and $+12 \mathrm{BHO}\left(d_{\text {coat }}=30 \mathrm{~nm}\right)$ films at 77,65 and $26 \mathrm{~K}$, respectively. For comparison, the data for $\mathrm{Ba}_{2} \mathrm{YNbO}_{6} \mathrm{NR}-\mathrm{doped}$ PLD films, ${ }^{45} \mathrm{BaZrO}_{3}$ (BZO) NR-doped PLD films, ${ }^{47}$ BZO nanoparticle (NP)-doped metal organic deposition (MOD) films ${ }^{21}$ and BZO NR-doped MOCVD films ${ }^{17}$ are included.

Table 1 Critical current density ratio at $3 \mathrm{~T}$

\begin{tabular}{cccc}
\hline Material & \multicolumn{3}{c}{$J_{c, \min }^{\mathrm{BHO}} / J_{c, \min }^{(\mathrm{YGd}) \mathrm{BCO}}$ at 3 T } \\
\hline \\
$+12 \mathrm{BZO}\left(d_{\text {coat }}=150 \mathrm{~nm}\right)$ & 1.5 & $65 \mathrm{~K}$ & $77 \mathrm{~K}$ \\
$+12 \mathrm{BHO}\left(d_{\text {coat }}=30 \mathrm{~nm}\right)$ & 6.2 & 2.4 & 2.7 \\
\hline
\end{tabular}

The $J_{c}$ ratio $\left(=J_{c, \text { min }}^{\mathrm{BHO}} / J_{c, \text { min }}^{(\mathrm{YGd})}\right)$ for $+12 \mathrm{BZO}\left(d_{\text {coat }}=150 \mathrm{~nm}\right)$ and $+12 \mathrm{BHO}\left(d_{\text {coat }}=30 \mathrm{~nm}\right)$ films at $3 \mathrm{~T}$ and different temperatures.

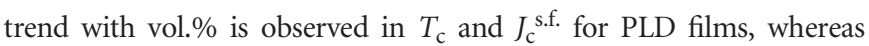
for MOD-BHO-NPs, $T_{\mathrm{c}}$ is constant and $J_{\mathrm{c}}$ s.f. increases. The trends of the $c$-axis expansion and $T_{\mathrm{c}}$ decrease in PLD films are consistent with oxygen-deficient regions surrounding the inclusions. ${ }^{39}$ Highresolution plan-view images and geometrical phase analysis (Figures $3 \mathrm{~d}$ and e) show the strain induced by PLD-NRs and MOD-NPs in the matrix. The strain component maps show that tensile and compressive stain are localized around the coherent NR interfaces and extend for more than $10 \mathrm{~nm}$ that has been observed in other PLD-BZO-NRs films. ${ }^{39,40}$ The $>10 \mathrm{~nm}$ extended strain region should lead to NRs overlapping at low dopant vol.\% with a corresponding disruption of current percolation and lower $T_{\mathrm{c}}$ and $J_{\mathcal{c}}^{\text {s.f. }}$. For the MOD-BHO-NPs, the strain is localized $(\sim 2 \mathrm{~nm})$ around the incoherent $\mathrm{NP} /$ matrix interfaces and decays to strain fluctuation values typical of undoped MOD films. The small strained regions leave the matrix unaltered with no degradation of the superconducting properties. The sharp interfaces also have a favorable effect on the pinning force; for a similar pinning energy, a smaller region of variation produces a larger pinning force. To summarize, the key factors for enhancing current transport are a small NP size ( $\sim$ comparable to the vortex core size), high NP density and a sharp NP interface with the matrix.

Dramatically higher $J_{c}$ with nearly isotropic angular dependence We now focus on the in-field properties. In Figures $4 a-c$, we show the field dependence of $J_{\mathrm{C}}\left(\mathbf{H} \|_{\mathbf{c}}\right)$ at different temperatures for the standard $+12 \mathrm{BZO}\left(d_{\text {coat }}=150 \mathrm{~nm}\right)$ and $+12 \mathrm{BHO}\left(d_{\text {coat }}=30 \mathrm{~nm}\right)$ films. For the standard film, $J_{\mathrm{c}}$ is nearly constant at low fields up to a characteristic crossover field $\mu_{0} H^{\star}$, defined at a criterion of $0.9^{\star} J_{\mathcal{C}}$ s.f. , followed by a power-law regime $\left(J_{c} \propto H^{-\alpha}\right)$ at intermediate fields. Finally, a more 
a
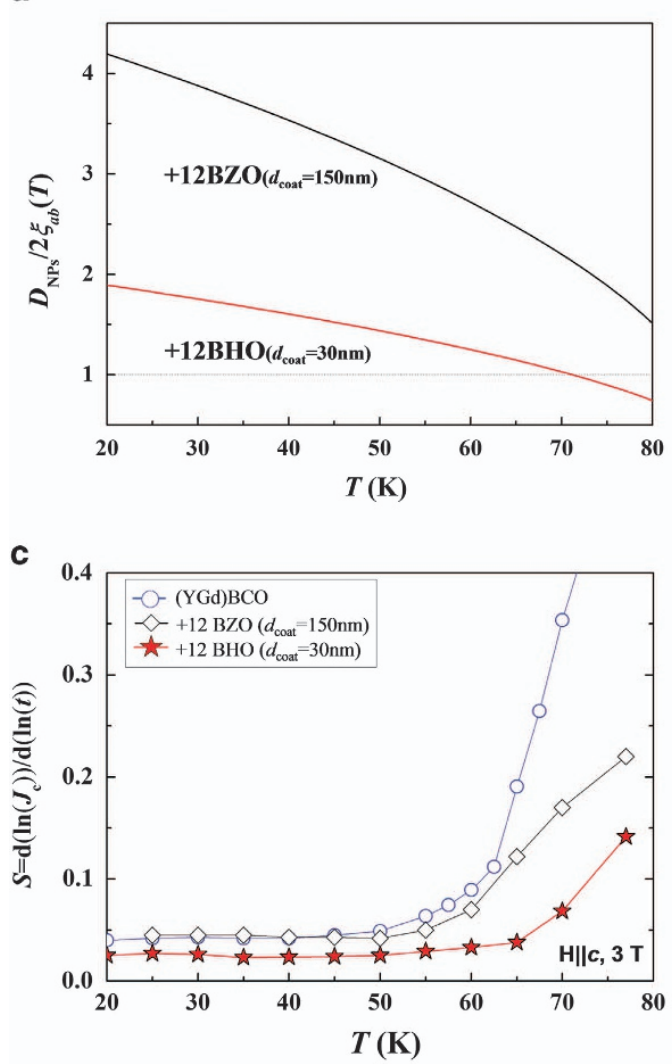

b

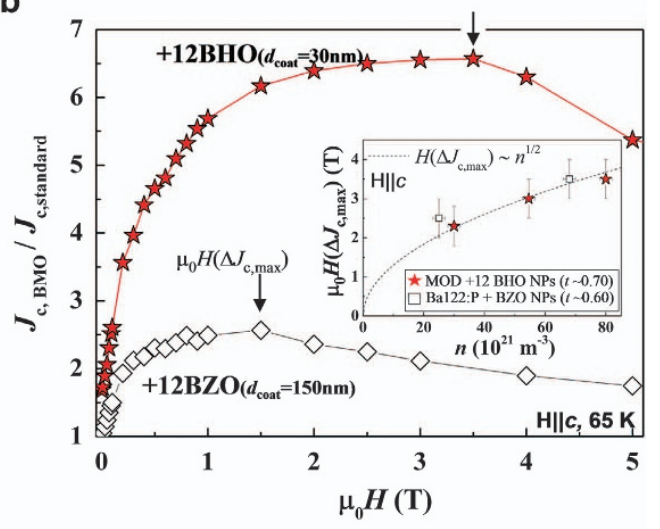

d

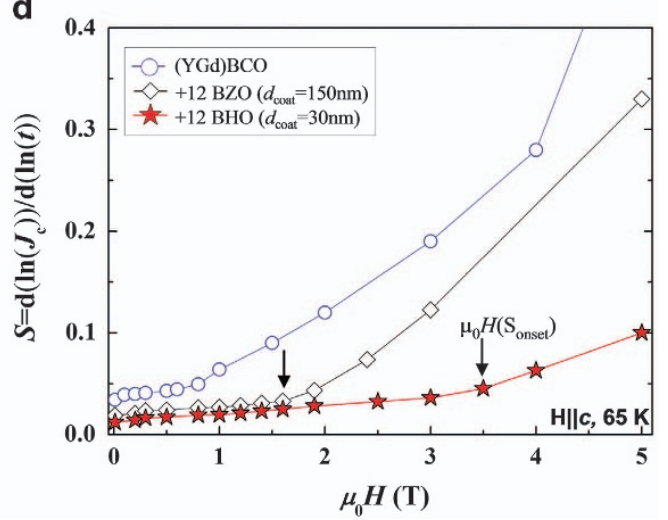

Figure 5 Field dependence of the flux creep rate and the $J_{\mathrm{C}}$ ratio. (a) Temperature dependence of the size ratio $\left(D_{\mathrm{NPs}} / 2 \xi_{\mathrm{ab}}\right)$ for the $+12 \mathrm{BZO}\left(d_{\mathrm{coat}}=150 \mathrm{~nm}\right)$ and $+12 \mathrm{BHO}\left(d_{\text {coat }}=30 \mathrm{~nm}\right)$ films. (b) Field versus $J_{\mathrm{C}}$ ratio $\left(\Delta J_{\mathrm{C}}=J_{c, \mathrm{BMO}} / J_{c \text {, standard }}\right)$ at $\mathbf{H} \| \mathrm{C}$ and $65 \mathrm{~K}$ for $+12 \mathrm{BZO}$ ( $\left.d_{\text {coat }}=150 \mathrm{~nm}\right)$ and $+12 \mathrm{BHO}\left(d_{\text {coat }}=30 \mathrm{~nm}\right)$ films. Inset: $H\left(\Delta J_{c, \text { max }}\right)$ at $\mathbf{H} \| c$ for $\mathrm{BaZrO}_{3}$ (BZO) nanoparticle (NP)-doped iron-based films 44 (open squares); and for $+12 \mathrm{BHO}$ $\left(d_{\text {coat }}=30 \mathrm{~nm}\right)$ films (solid stars). $H\left(\Delta J_{c, \max }\right)$ at HIlc. (c) Temperature dependence of $S$ for the $(\mathrm{YGd}) \mathrm{BCO},+12 \mathrm{BZO}\left(d_{\text {coat }}=150 \mathrm{~nm}\right)$ and $+12 \mathrm{BHO}$ $\left(d_{\text {coat }}=30 \mathrm{~nm}\right)$ films at $\mathbf{H} \| \mathrm{C}$ and $3 \mathrm{~T}$. (d) Field dependence of the flux creep rate $S$ for the $(\mathrm{YGd}) \mathrm{BCO},+12 \mathrm{BZO}\left(d_{\text {coat }}=150 \mathrm{~nm}\right)$ and $+12 \mathrm{BHO}\left(d_{\text {coat }}=30 \mathrm{~nm}\right)$ films at $\mathrm{HIl}$ and $65 \mathrm{~K}$.

rapid decay of $J_{\mathrm{c}}$ is observed as $H$ approaches the irreversibility field. This behavior occurs at all temperatures with $\alpha \sim 0.63-0.7 .^{41}$

For both NP films, we observe an increase in $J_{\mathcal{c}}^{\text {s.f. }}$ and $\mu_{0} H^{\star}$ at all temperatures, consistent with a much larger density of NPs. ${ }^{42}$ This improvement is maintained as a function of field with $J_{c}(H)$ that does not follow the standard power-law dependence. This type of non-power-law dependence is similar to that observed in cuprate $^{21,31,41,43}$ and pnictide films ${ }^{44}$ with the strong pinning in both cases being due to the NPs. Because of the large NP size $(\sim 25 \mathrm{~nm})$ in the $+12 \mathrm{BZO}\left(d_{\text {coat }}=150 \mathrm{~nm}\right)$ film, NPs cease being the dominant pinning center at low temperatures; the significant improvements at high temperatures are lost, and a power-law dependence is regained similarly to the reference film. ${ }^{41}$ However, given the small size $(\sim 7 \mathrm{~nm})$ of the $+12 \mathrm{BHO}\left(d_{\text {coat }}=30 \mathrm{~nm}\right)$ films, a non-power-law $J_{\mathrm{c}}$ $(H)$ decay is observed, indicating that NPs are the dominant pinning center at all temperatures. This is also seen in the magnitude of the $J_{\mathrm{c}}$ increase at $3 \mathrm{~T}$ that is approximately a factor of 6 at all temperatures (see Table 1).

The remarkable in-field performance of the $+12 \mathrm{BHO}$ $\left(d_{\text {coat }}=30 \mathrm{~nm}\right)$ film is highlighted in the insets of Figures $4 \mathrm{a}-\mathrm{c}$, where the pinning force, $F_{\mathrm{p}}=J_{\mathrm{c}}(H) \times \mu_{0} H$, is compared with the highest previously reported values. ${ }^{34,45}$ At high temperatures, the $+12 \mathrm{BHO}$ $\left(d_{\text {coat }}=30 \mathrm{~nm}\right)$ film shows a maximum $F_{\mathrm{p}}=33 \mathrm{GN} \mathrm{m}^{-3}$ at $77 \mathrm{~K}$ and $124 \mathrm{GN} \mathrm{m}^{-3}$ at $65 \mathrm{~K}$. Moreover, at $T=26 \mathrm{~K}$, the $F_{\mathrm{p}}$ of the $+12 \mathrm{BHO}$ $\left(d_{\text {coat }}=30 \mathrm{~nm}\right)$ film reaches a nearly constant $\sim 677 \mathrm{GN} \mathrm{m}^{-3}$ from $\mu_{0} H>3 \mathrm{~T}$ up to $9 \mathrm{~T}$, an $F_{\mathrm{p}}$ value 10 times greater than that of $\mathrm{Nb}_{3} \mathrm{Sn}$ at $4.2 \mathrm{~K}^{46}$ Because the pinning landscape is produced by NPs, $F_{\mathrm{p}}$ measured along the $c$-axis is actually close to the minimum value as a function of angle, not the maximum, contrary to the case of films with NRs, ${ }^{45}$ as can be observed in the $J_{\mathrm{c}}(\theta)$ curves of Figures $4 \mathrm{~d}-\mathrm{f}$. For the $+12 \mathrm{BZO}\left(d_{\text {coat }}=150 \mathrm{~nm}\right)$ film, the BZO NPs are much less effective at $26 \mathrm{~K}$ than at a higher $T$. However, for the $+12 \mathrm{BHO}$ $\left(d_{\text {coat }}=30 \mathrm{~nm}\right)$ film, we see significant $J_{\mathrm{c}}$ increases for all orientations and temperatures compared with that of the BZO MOD films, with a higher $J_{c}$ minimum $\left(J_{c, \min }\right)$ than that previously reported for any REBCO film or wire. ${ }^{17,21,45,47,48}$

\section{Relation between flux creep and NP density}

The different temperature dependence of the $J_{\mathrm{c}}$ improvement (seen in the $J_{\mathrm{c}}$ ratio) between $\mathrm{BZO}$ and $\mathrm{BHO}$ has its origin in the NP size $\left(D_{\mathrm{NP}}\right)$ relative to the normal-core diameter $\left(2 \xi_{a b}\right)$, as shown in Figure 5a. For BZO $\left(D_{\mathrm{NP}} \sim 25 \mathrm{~nm}\right), D_{\mathrm{NP}} / 2 \xi_{a b}$ is 1.5 at $80 \mathrm{~K}$ but $>4$ at low temperatures, whereas for $\mathrm{BHO}\left(D_{\mathrm{NP}} \sim 7 \mathrm{~nm}\right), d / 2 \xi_{a b} \leqslant 1$ and $\sim 2$ at high and low temperatures, respectively. Values of $d / 2 \xi_{a b}>3$ or $\ll<1$ lead to less effective pinning; ${ }^{44}$ thus, the ability of the $7-\mathrm{nm}$ BHO NPs to increase $J_{\mathrm{c}}$ is higher and is restrained to lower temperatures.

Insights into the benefit of further increasing NP density are found by plotting the ratio $J_{\mathrm{c}, \mathrm{BMO}} / J_{\mathrm{c} \text {,standard }}$ vs $\mu_{0} H$ in Figure $5 \mathrm{~b}$; this ratio has 
a

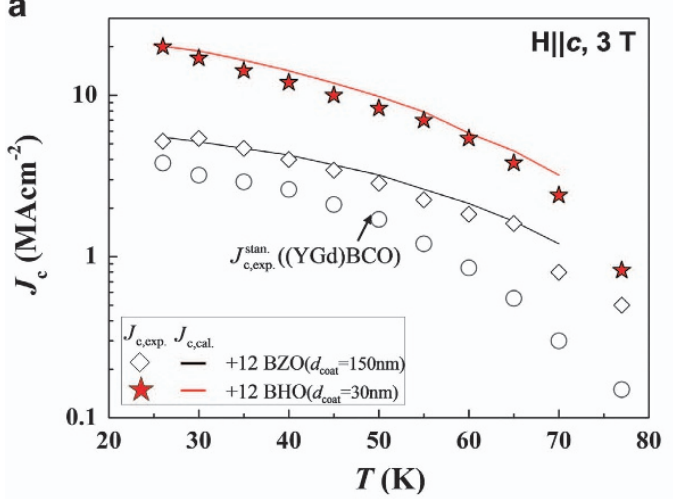

c

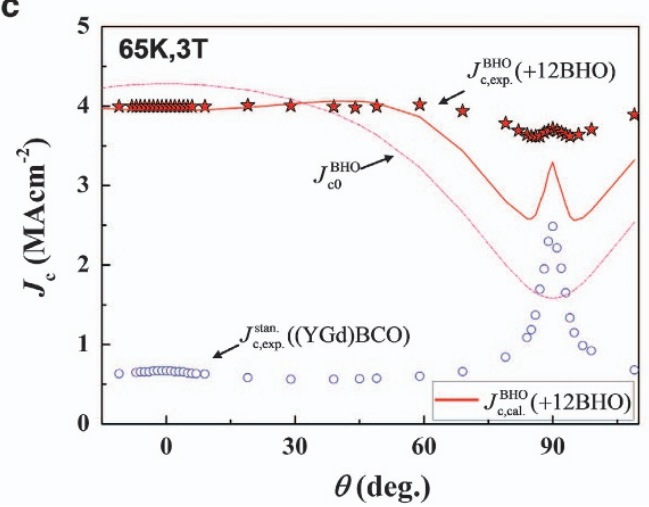

b

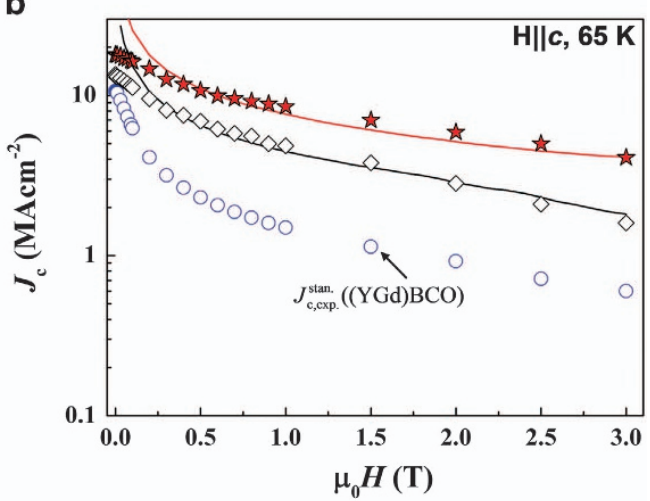

d

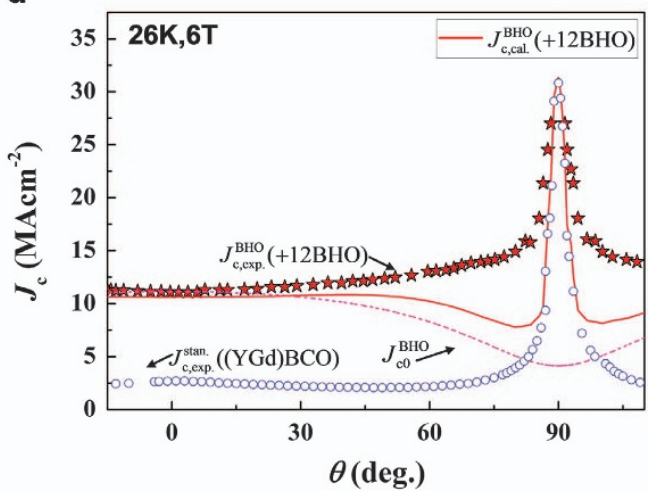

Figure 6 Experimental and theoretical $J_{C}$ as a function of field, temperature and angle. (a) Temperature dependence of the experimental $J_{C}$ (symbols) and theoretical $J_{c}$ (solid lines) for $+12 \mathrm{BZO}\left(d_{\text {coat }}=150 \mathrm{~nm}\right)$ and $+12 \mathrm{BHO}\left(d_{\text {coat }}=30 \mathrm{~nm}\right)$ films at H\|lc and $65 \mathrm{~K}$. (b) The field dependence of experimental $J_{c}$ (symbols) and theoretical $J_{c}$ (solid lines) for $+12 \mathrm{BZO}\left(d_{\text {coat }}=150 \mathrm{~nm}\right)$ and $+12 \mathrm{BHO}\left(d_{\text {coat }}=30 \mathrm{~nm}\right)$ films at $\mathrm{H} \| \mathrm{C}$ and $65 \mathrm{~K}$. (c) d) Angle dependence of experimental $J_{C}$ (symbols) and theoretical $J_{C}$ (solid lines for $(Y G d) B C O,+12 B Z O\left(d_{\text {coat }}=150 \mathrm{~nm}\right)$ and $+12 \mathrm{BHO}\left(d_{\text {coat }}=30 \mathrm{~nm}\right)$ films at $\mathbf{H} \| \mathrm{C}$ and $65 \mathrm{~K}$. In $(\mathbf{c}, \mathbf{d})$, the dashed line is the $J_{\mathrm{cO}}$ for the $+12 \mathrm{BHO}\left(d_{\text {coat }}=30 \mathrm{~nm}\right)$ films at each measurement condition. For comparison, the experimental $J_{\mathrm{c}}$ of $(Y \mathrm{YG}) \mathrm{BCO}$ films is also shown in (a-d).

a strong correlation with the density of NPs. ${ }^{44}$ The $+12 \mathrm{BHO}$ $\left(d_{\text {coat }}=30 \mathrm{~nm}\right)$ film shows a maximum $J_{\mathrm{c}}$ ratio $\left(\Delta J_{\mathrm{c}, \max }\right)$ of 6.5 near $\mu_{0} H\left(\Delta J_{c, \max }\right) \sim 3.5 \mathrm{~T}$. The field values are extremely close to $\mu_{0} H_{\mathrm{NP}}$, the field at which the intervortex distance $\left(a_{\mathrm{f}}\right)$ matches $L_{\mathrm{NP}}(\sim 20 \mathrm{~nm})$. The new data fit extremely well with the $\mu_{0} H\left(\Delta J_{c, \text { max }}\right) \sim n^{\sim 1 / 2}$ universal dependence ${ }^{44}$ we found for the cuprates and pnictides with NPs, indicating that the maximum effects are obtained when $a_{\mathrm{f}} \sim L_{\mathrm{NP}}$.

This condition $\left(a_{\mathrm{f}} \sim L_{\mathrm{NP}}\right)$ is also manifested in the flux creep rate. The flux creep rate $S=-\mathrm{d} \ln \left(J_{c}\right) / \mathrm{d} \ln (t)$ measures the rate at which vortices exit pinning centers as a result of fluctuations. These fluctuations enable vortex depinning via different types of excitations, thus effectively reducing $J_{\mathrm{c}} \cdot{ }^{49}$ In Figure $5 \mathrm{~d}$, we find that $S$ is significantly reduced with respect to that of $(\mathrm{Y}, \mathrm{Gd}) \mathrm{BCO}$ when NPs are included. The stronger the pinning, the smaller $S$ is; however, more importantly, the reduction of $S(H)$ continues up to higher field strengths with an increasing density of BMO NPs; the maximum field for the low creep onset $\left(\mu_{0} H\left(S_{\text {onset }}\right)\right.$ ) is $1.5 \mathrm{~T}$ for the $+12 \mathrm{BZO}$ $\left(d_{\text {coat }}=150 \mathrm{~nm}\right)$ film and $3.5 \mathrm{~T}$ for the $+12 \mathrm{BHO}\left(d_{\text {coat }}=30 \mathrm{~nm}\right)$ film (see arrows in Figure 5b). Thus, it is clear that the maximum improvement, as evidenced by $\mu_{0} H\left(\Delta J_{c, \max }\right)$ and $\left.\mu_{0} H\left(S_{\text {onset }}\right)\right)$, can be achieved with the same characteristic field when $a_{\mathrm{f}} \sim L_{\mathrm{NP}}$, where $a_{\mathrm{f}}$ is the intervortex distance.

Not only is the onset pushed to greater fields, but the field slope of $S$ is greatly reduced, such that $S$ for $\mathrm{BHO}$ at large applied fields is much smaller. This reduction in $S$ is maintained at all temperatures, as shown in Figure 5c. There is additional evidence of the link between $S$ improvement and $J_{c}$ enhancement; a lower $S(T)$ for BZO is observed down to $\sim 40 \mathrm{~K}$, where $D_{\mathrm{BZO}} / 2 \xi_{a b}(T) \sim 3$, which is concomitant with the loss of the non-power-law behavior in $J_{\mathrm{c}}(H)$ and the marked decrease in the $J_{\mathrm{c}, \mathrm{BZO}} / J_{\mathrm{c}, \text { standard }}$ ratio. ${ }^{41}$ On the other hand, the $+12 \mathrm{BHO}\left(d_{\text {coat }}=30 \mathrm{~nm}\right)$ film shows a lower $S$ at all temperatures compared with that of the other two films. These data indicate the importance of both density and size to reduce flux creep as well as to increase $J_{c}$. Ultimately, the maximum improvement produced by the $\mathrm{NP}$ additions in $J_{\mathrm{c}}(H)$ occurs when the elastic energy is minimized and thermal activation effects are reduced by the strong pinning of the high-density BHO NPs.

All of these improvements are obtained with simple multicoating deposition without the need for ex situ treatments or convoluted procedures. The easily controlled and fabricated nanostructured functional material with greatly enhanced and nearly isotropic $J_{\mathrm{c}}(\theta)$ shows that materials can be grown to target different applications.

Simple pinning model for superconductor with strong pinning NPs We have seen the clear effect of high-density BHO NPs on the enhancement of $J_{c}$ and the reduction of thermal activation. The challenge now is to quantify improvements to be able to target specific applications based on $J_{c}(T, H, \theta)$. Several models have been proposed to explain $J_{\mathrm{c}}(T, H, \theta)$ in cuprate and pnictide superconductors with strong pinning NPs or NRs. ${ }^{21,24,50-59}$ Although the influence of creep 
on $J_{c}$ for these superconductors has been known for a long time, ${ }^{49,60}$ only a few attempts have been made to link the pinning improvements to $J_{c}$ and $S^{60,61}$

To calculate the temperature, magnetic field and field angle dependence of $J_{c}\left(J_{c, \text { calc. }}\right)$, we use $J_{c, \text { calc. }}(T, H, \theta)=$ $J_{c 0}(T, H, \theta)\left(1-S \ln \left(t / t_{0}\right)\right)$, where $t_{0}$ is the effective attempt time, $J_{\mathrm{c} 0}$ is the creep-free $J_{\mathrm{c}}$ and $S$ is the experimentally obtained flux creep rate.

Here, we assume that NPs are elliptical; thus, the creep-free $J_{\mathrm{c}}$ for NPs is given by

$$
\begin{aligned}
J_{\mathrm{c} 0}^{\mathrm{NPs}}(T, \theta, H)= & n_{\mathrm{np}}\left(\frac{\left(\mu_{0} H_{\mathrm{c}}(T)\right)^{2} \pi \xi_{a b}(T) l_{0}}{4 \mu_{0}}\right) \\
& \left(\frac{\cos ^{2} \theta+\gamma^{-2} \sin ^{2} \theta}{\cos ^{2} \theta+(c / b)^{2} \sin ^{2} \theta}\right)^{0.5}\left(1-\frac{T}{T_{\mathrm{c}}}\right)^{1.5} \\
& \left(\mu_{0} H\right)^{-0.5}\left(1-\frac{\mu_{0} H}{\mu_{0} H_{\mathrm{c} 2}(T)}\right)^{2}
\end{aligned}
$$

where $l_{0}$ is the NP diameter at $\theta=0^{\circ}$, that is, along the $c$ orientation, $c / b$ is the ratio of the NP dimensions along the $c$ and $a b$ orientations, respectively, $\varepsilon(\theta)=\left[\cos ^{2}(\theta)+\gamma^{-2} \sin ^{2}(\theta)\right]^{0.5}$ and $\gamma$ is the electronic mass anisotropy. ${ }^{50}$ Here, the temperature dependencies are simply assumed as $H_{\mathrm{c}}(T)=H_{\mathrm{c}}(0)\left(1-T / T_{\mathrm{c}}\right)$ and $\xi_{\mathrm{ab}}(T)=$ $\xi_{\mathrm{ab}}(0)\left(1-T / T_{\mathrm{c}}\right)^{-0.5}$, and the field-dependent factor $\left(1-\mathrm{H} / \mathrm{H}_{\mathrm{c} 2}\right)^{2}$ comes from the reduction in the condensation energy. ${ }^{62}$ A detailed derivation of these equations is shown in Supplementary Figure S1. We focus on the additional pinning from NPs, assuming that the rest of the pinning landscape remains unchanged. To take into account the effect of fluctuations, $J_{\mathrm{c} 0}$ (creep-free $J_{\mathrm{c}}$ ) must be calculated that is then added to the NP contribution using statistical summation. ${ }^{63,64}$ Using $J_{c 0}^{\text {stan. }}(T, H, \theta)=J_{c \text {,exp. }}^{\text {stan. }}(T, H, \theta) /\left(1-S^{\text {stan. }} \cdot \ln \left(t / t_{0}\right)\right)$, where $J_{c, \text { exp. }}^{\text {stan. }}(T, H, \theta)$ is the experimental $J_{c}$ for standard $(\mathrm{Y}, \mathrm{Gd}) \mathrm{BCO}$, the calculated $J_{\mathrm{c}}$ for a composite sample is

$$
J_{c, \text { cal. }}^{\mathrm{BHO}}(T, H, \theta)=\left[J_{\mathrm{c} 0}^{\mathrm{stan} .}(T, H, \theta)^{2}+J_{\mathrm{c} 0}^{\mathrm{NPs}}(T, H, \theta)^{2}\right]^{0.5}\left(1-S^{\mathrm{BHO}} \ln \left(t / t_{0}\right)\right)
$$

The parameters used are listed in Supplementary Table S1.

Figure 6a shows the temperature dependence of the experimental $J_{\mathrm{c}}$ (symbols) and calculated $J_{c}$ (solid lines) for the $+12 \mathrm{BZO}$ $\left(d_{\text {coat }}=150 \mathrm{~nm}\right)$ and $+12 \mathrm{BHO}\left(d_{\text {coat }}=30 \mathrm{~nm}\right)$ films at $\mathbf{H} \|_{c}=3 \mathrm{~T}$. Using as average NP size $D_{\mathrm{BMO}}$ from microstructural data and the experimental $S(T)$, in Figure 6a, we show that the calculated $J_{c}$ values for both films are in good agreement with the experimental $J_{c}$. The field dependence of the calculated $J_{c}$ at $\mathbf{H} \|_{c}$ and $65 \mathrm{~K}$ for NP-doped films is again in very good agreement with the experimental $J_{\mathrm{C}}(T)$ data shown in Figure $6 \mathrm{~b}$. Figures $6 \mathrm{c}$ and $\mathrm{d}$ show the angular dependences of the measured $J_{\mathrm{c}}$ (symbols) and the calculated $J_{\mathrm{c}}$ (lines) for the $+12 \mathrm{BHO}\left(d_{\text {coat }}=30 \mathrm{~nm}\right)$ film at $(65 \mathrm{~K}, 3 \mathrm{~T})$ and $(26 \mathrm{~K}, 6 \mathrm{~T})$ and the calculated $J_{\mathrm{c} 0}$ for the $\mathrm{BHO} \mathrm{NPs}\left(J_{\mathrm{c} 0}^{\mathrm{BHO}}\right)$. The agreement is very satisfactory in both cases given that the large temperature difference ( 65 and $26 \mathrm{~K}$ ) induces large changes in the superconducting values that are taken into account by the model using the same parameter values. Careful comparison of the experimental and calculated $J_{\mathrm{c}}(\theta)$ show some disagreement at $\sim 30-80^{\circ}$ with better fits for BZO (Supplementary Figure S2) than BHO (Figure 6). The deviation is associated with the NP contribution and could be associated with only using the average value of the NP size rather than the actual size distribution and because of the fact that the pinning landscape changes slightly after adding NPs. ${ }^{26}$ The most important feature of the present model is that all the parameters used are derived by fitting to a limited set of experimental data (no free parameters) and that temperature, angle and field properties at other experimental conditions are then calculated.

\section{CONCLUSION}

To summarize, we have demonstrated the ability of adding a large density of second-phase NPs of tunable size without affecting the host matrix. We show that limiting diffusion (thermally and/or spatially) is the key to reducing the NP size; we achieve this by selecting a secondphase material with lower crystallization temperature and modulating the precursor composition through the deposition layer thickness. This ability, coupled with extremely good modeling, indicates that MOD REBCO can be used for specific performance requirements and that this NP tuning method is amenable to other functional perovskite materials.

\section{CONFLICT OF INTEREST}

The authors declare no conflict of interest.

\section{ACKNOWLEDGEMENTS}

This work was supported by the Laboratory Directed Research and Development program at Los Alamos National Laboratory (to MM) and by the US Department of Energy, Office of Basic Energy Sciences, Materials Sciences and Engineering Division (to BM). MM is supported by JSPS KAKENHI (17H03239 and 17K18888). MM and BM thank Jeffrey O Willis for helpful discussions and a critical reading of the manuscript. MM also thank Akira Ibi for sample fabrication of $\mathrm{BaHfO}_{3}$ nanorods doped PLD-GdBCO films.

Author contributions: MM grew the films, carried out the experimental design, transport measurements, flux creep measurements and prepared the manuscript. BM carried out data analysis and provided advice and consultation on flux pinning and manuscript preparation. MS and MoK processed the films and performed the transport measurements. TaK and ToK performed the microstructural studies. TI provided advice and consultation on film preparation. SA and PM contributed to discussion on flux pinning. MaK and TM provided advice and consultation on theoretical critical current calculation. All authors discussed the results and implications and commented on the manuscript.

\section{PUBLISHER'S NOTE}

Springer Nature remains neutral with regard to jurisdictional claims in published maps and institutional affiliations.

1 Hu, X., Jood, P., Ohta, M., Kunii, M., Nagase, K., Nishiate, H., Kanatzidis, M. G. \& Yamamoto, A. Power generation from nanostructured PbTe-based thermoelectrics: comprehensive development from materials to modules. Energy Environ. Sci. 9, 517 (2016).

2 Ichino, Y., Yamazaki, K., Yoshikawa, T., Yoshida, Y. \& Takai, Y. Thermoelectric properties of nano-structure controlled $\mathrm{Sm} 2-x \mathrm{CexCuO} 4$ thin films. Mater. Res. Soc. Symp. Proc. 928, 0928-GG14-17 (2006).

3 Foltyn, S. R., Civale, L., Macmanus-Driscoll, J. L., Jia, Q. X., Maiorov, B., Wang, H. \& Maley, M. Materials science challenges for high-temperature superconducting wire. Nat. Mater. 6, 631-642 (2007).

4 MacManus-Driscoll, J. L., Foltyn, S. R., Jia, Q. X., Wang, H., Serquis, A., Civale, L., Maiorov, B., Hawley, M. E., Maley, M. P. \& Peterson, D. E. Strongly enhanced current densities in superconducting coated conductors of $\mathrm{YBa}_{2} \mathrm{Cu}_{3} \mathrm{O}_{7-x}+\mathrm{BaZrO}_{3}$. Nat. Mater. 3, 439-443 (2004)

5 Hicks, L. D. \& Dresselhaus, M. S. Effect of quantum-well structures on the thermoelectric figure of merit. Phys. Rev. B 47, 12727 (1993).

6 Venkatasubramanian, R., Siivola, E., Colpitts, T. \& O'Quinn, B. T Thin-film thermoelectric devices with high room-temperature figures of merit. Nature 413 , 597 (2001).

7 Ohta, H., Kim, S., Mune, Y., Mizoguchi, T., Nomura, K., Ohta, S., Nomura, T., Nakanishi, Y., Ikuhara, Y., Hirano, M., Hosono, H. \& Koumoto, K. Giant thermoelectric Seebeck coefficient of a two-dimensional electron gas in $\mathrm{SrTiO}_{3}$. Nat. Mater. 6, 129-134 (2007). 
8 Loureiro, J., Neves, N., Barros, R., Mateus, T., Santos, R., Filonovich, S., Reparaz, S., Sotomayor-Torres, C. M., Wyczisk, F., Divay, L., Martins, R. \& Ferreira, I. Transparent aluminium zinc oxide thin films with enhanced thermoelectric properties. J. Mater. Chem. A 2, 6649-6655 (2014).

9 Matsumoto, O., Utsuki, A., Tsukada, A., Yamamoto, H., Manabe, T. \& Naito, M Synthesis and properties of superconducting $\mathrm{T}^{\prime}-\mathrm{R}_{2} \mathrm{CuO}_{4}(\mathrm{R}=\mathrm{Pr}, \mathrm{Nd}, \mathrm{Sm}, \mathrm{Eu}, \mathrm{Gd})$. Phys. Rev. B 79, 100508(R) (2009).

10 Wang, Y., Lee, K. H., Hyuga, H., Kita, H., Ohta, H. \& Koumoto, K. Enhancement of thermoelectric performance in rare earth-doped $\mathrm{Sr}_{3} \mathrm{Ti}_{2} \mathrm{O}_{7}$ by symmetry restoration of $\mathrm{TiO}_{6}$ octahedra. J. Electroceram. 24, 76 (2010).

11 Kawazoe, H., Yasukawa, M., Hyodo, H., Kurita, M, Yanagi, H. \& Hosono, H. P-type electrical conduction in transparent thin films of $\mathrm{CuAlO}_{2}$. Nature 389, 939 (1997).

12 Haugan, T., Barnes, P. N., Wheeler, R., Meisenkothen, F. \& Sumption, M. Addition of nanoparticle dispersions to enhance flux pinning of the $\mathrm{YBa}_{2} \mathrm{Cu}_{3} \mathrm{O}_{7-x}$ superconductor. Nature 430, 867-870 (2004).

13 Kang, S., Goyal, A., Li, J., Gapud, A. A., Martin, P. M., Heatherly, L., Thompson, J. R., Christen, D. K., List, F. A., Paranthaman, M. \& Lee, D. F. High-performance high- $T_{\mathrm{c}}$ superconducting wires. Science 311, 1911-1914 (2006).

14 Matsumoto, K. \& Mele, P. Artificial pinning center technology to enhance vortex pinning in YBCO coated conductors. Supercond. Sci. Technol. 23, 014001 (2010).

15 Opherden, L., Sieger, M., Pahlke, P., Hühne, R., Schultz, L., Meledin, A., Tendeloo, G. V., Nast, R., Holzapfel, B., Marco, B., MacManus-Driscoll, J. L. \& Hänisch, J. Large pinning forces and matching effects in $\mathrm{YBa}_{2} \mathrm{Cu}_{3} \mathrm{O}_{7-\delta}$ thin films with $\mathrm{Ba}_{2} \mathrm{Y}(\mathrm{Nb} / \mathrm{Ta}) \mathrm{O}_{6}$ nanoprecipitates. Sci. Rep. 6, 21188 (2016).

16 Cayado, P., Keukeleere, K. D., Garzón, A., Perez-Mirabet, L., Meledin, A., Roo, J. D., Vallés, F., Mundet, B., Rijckaert, H. \& Pollefeyt, G. Epitaxial $\mathrm{YBa}_{2} \mathrm{Cu}_{3} \mathrm{O}_{7-x}$ nanocomposite thin films from colloidal solutions. Supercond. Sci. Technol. 28, 124007 (2015)

17 Selvamanickam, V., Xu, A., Liu, Y., Khatri, N. D., Lei, C., Chen, Y., Galstyan, E. \& Majkic, G. Correlation between in-field critical currents in $\mathrm{Zr}$-added ( $\mathrm{Gd}, \mathrm{Y}) \mathrm{Ba}_{2} \mathrm{Cu}_{3} \mathrm{O}_{x}$ superconducting tapes at 30 and 77 K. Supercond. Sci. Technol. 27, 055010 (2014).

18 Xu, A., Delgado, L., Khatri, N., Liu, Y., Selvamanickam, V., Abraimov, D., Jaroszynski, J., Kametani, F. \& Larbalestier, D. C. Strongly enhanced vortex pinning from 4 to $77 \mathrm{~K}$ in magnetic fields up to $31 \mathrm{~T}$ in 15 mol.\% Zr-added (Gd, Y)-Ba-Cu-O superconducting tapes. APL Mater 2, 046111 (2014).

19 Selvamanickam, V., Gharahcheshmeh, M H., Xu, A., Zhang, Y. \& Galstyan, E. Critical current density above $15 \mathrm{MAcm}^{-2}$ at $30 \mathrm{~K}, 3 \mathrm{~T}$ in $2.2 \mu \mathrm{m}$ thick heavily-doped (Gd,Y) $\mathrm{Ba}_{2} \mathrm{Cu}_{3} \mathrm{O}_{\mathrm{x}}$ superconductor tapes. Supercond. Sci. Technol. 28, 072002 (2015).

20 Miura, S., Yoshida, Y., Ichino, Y., Matsumoto, K., Ichinose, A. \& Awaji, S. Characteristics of high-performance $\mathrm{BaHfO}_{3}$-doped $\mathrm{SmBa}_{2} \mathrm{Cu}_{3} \mathrm{O}_{y}$ superconducting films fabricated with a seed layer and low temperature growth. Supercond. Sci. Technol. $\mathbf{2 8}$ 065013 (2015)

21 Gutiérrez, J., Llordés, A., Gázquez, J., Gibert, M., Romà, N., Ricart, S., Pomar, A. Sandiumenge, F., Mestres, N., Puig, T. \& Obradors, X. Strong isotropic flux pinning in $\mathrm{YBa}_{2} \mathrm{Cu}_{3} \mathrm{O}_{7-x}+\mathrm{BaZrO}_{3}$ films derived from chemical solutions. Nat. Mater. 6, 367-373 (2007)

22 Engel, S., Thersleff, T., Hühne, R., Schultz, L. \& Holzapfel, B. Enhanced flux pinning in $\mathrm{YBa}_{2} \mathrm{Cu}_{3} \mathrm{O}_{7}$ layers by the formation of nanosized $\mathrm{BaHfO}_{3}$ precipitates using the chemical deposition method. Appl. Phys. Lett. 90 102505 (2007).

23 Llordés, A., Palau, A., Gázquez, J., Coll, M., Vlad, R., Pomar, A., Arbiol, J., Guzmán, R., Ye, S., Rouco, V., Sandiumenge, F., Ricart, S., Puig, T., Varela, M., Chateigner, D., Vanacken, J., Gutiérrez, J., Moshchalkov, V., Deutscher, G., Magen, C. \& Obradors, X. Nanoscale strain-induced pair suppression as a vortexpinning mechanism in high temperature superconductors. Nat. Mater. 11, 329-336 (2012).

24 Palau, A., Bartolomé, E., Llordés, A., Puig, T. \& Obradors, X. Isotropic and anisotropic pinning in TFA-grown $\mathrm{YBa}_{2} \mathrm{Cu}_{3} \mathrm{O}_{7-x}$ films with $\mathrm{BaZrO}_{3}$ nanoparticles. Supercond. Sci. Technol. 24, 125010 (2011).

25 Weigand, M., Rutter, N. A. \& Durrell, J. H. In-plane critical current density measurements of straight and curved tracks in RABITS-MOD coated conductors. Supercond. Sci. Technol. 26, 105012 (2013).

26 Miura, M., Maiorov, B., Balakirev, F. F., Kato, T., Sato, M., Takagi, Y., Izumi, T. \& Civale, L. Upward shift of the vortex solid phase in high-temperature superconducting wires through high density nanoparticle addition. Sci. Rep. 6 , 20436 (2016)

27 Izumi, T. \& Shiohara, Y. R\&D of coated conductors for applications in Japan. Phys. C 470, 967-970 (2010)

28 Rupich, M. W., Li, X., Thieme, C., Sathyamurthy, S., Fleshler, S., Tucker, D., Thompson, E., Schreiber, J., Lynch, J. \& Buczek, D. Advances in second generation high temperature superconducting wire manufacturing and R\&D at American Superconductor Corporation. Supercond. Sci. Technol. 23, 014015 (2010).

29 Obradors, X. \& Puig, T. Coated conductors for power applications: materials challenges. Supercond. Sci. Technol. 27, 044003 (2014).

30 Miura, M., Maiorov, B., Willis, J. O., Kato, T., Sato, M., Izumi, T., Shiohara, Y. \& Civale, $\mathrm{L}$. The effects of density and size of $\mathrm{BaMO}_{3}(\mathrm{M}=\mathrm{Zr}, \mathrm{Nb}, \mathrm{Sn})$ nanoparticles on the vortex glassy and liquid phase in $(\mathrm{Y}, \mathrm{Gd}) \mathrm{Ba}_{2} \mathrm{Cu}_{3} \mathrm{O}_{y}$ coated conductors. Supercond. Sci. Technol. 26, 035008 (2013).
31 Maiorov, B., Baily, S. A., Zhou, H., Ugurlu, O., Kennison, J. A., Dowden, P. C., Holesinger, T. G., Foltyn, S. R. \& Civale, L. Synergetic combination of different types of defect to optimize pinning landscape using $\mathrm{BaZrO}_{3}$-doped $\mathrm{YBa}_{2} \mathrm{Cu}_{3} \mathrm{O}_{7}$. Nat. Mater. 8 398 (2009).

32 Horii, S., Kai, H., Mukaida, M., Yamada, K., Teranishi, R., Ichinose, A., Matsumoto, K., Yoshida, Y., Kita, R., Shimoyama, J. \& Kishio, K. Vortex Bose glass in $\mathrm{ErBa}_{2} \mathrm{Cu}_{3} \mathrm{O}_{y}$ films with size-controlled nanorods. Appl. Phys. Lett. 93, 152506 (2008).

33 Miura, M., Yoshizumi, M., Izumi, T. \& Shiohara, Y. Formation mechanism of $\mathrm{BaZrO}_{3}$ nanoparticles in $\mathrm{Y}_{1-x} \mathrm{Sm}_{x} \mathrm{Ba}_{2} \mathrm{Cu}_{3} \mathrm{O}_{y}$-coated conductors derived from trifluoroacetate metal-organic deposition. Supercond. Sci. Technol. 23, 014013 (2010).

34 Tsuruta, A., Yoshida, Y., Ichino, Y., Ichinose, A., Matsumoto, K. \& Awaji, S. The influence of the geometric characteristics of nanorods on the flux pinning in highperformance $\mathrm{BaMO}_{3}$-doped $\mathrm{SmBa}_{2} \mathrm{Cu}_{3} \mathrm{O}_{y}$ films $(\mathrm{M}=\mathrm{Hf}, \mathrm{Sn})$. Supercond. Sci. Technol. 27, 065001 (2014).

35 Wu, J. Z., Shi, J. J., Baca, J. F., Emergo, R., Haugan, T. J., Maiorov, B. \& Holesinger, T. The effect of lattice strain on the diameter of $\mathrm{BaZrO}_{3}$ nanorods in epitaxial $\mathrm{YBa}_{2} \mathrm{Cu}_{3} \mathrm{O}_{7}$ ${ }_{\delta}$ films.. Supercond. Sci. Technol. 27, 044010 (2014).

36 Brandt, E. H. Large range of validity of linear elasticity of the vortex lattice in high- $T_{c}$ superconductors. Phys. Rev. Lett. 69, 1105-1108 (1992).

37 Gurevich, A. Pinning size effects in critical currents of superconducting films. Supercond. Sci. Technol. 20, S128-S135 (2007).

38 Mele, P., Guzman, R., Gazquez, J., Puig, T., Obradors, X., Saini, S., Yoshida, Y., Mukaida, M., Ichinose, A., Matsumoto, K. \& Adam, M. I. High pinning performance of $\mathrm{YBa}_{2} \mathrm{Cu}_{3} \mathrm{O}_{7-\delta}$ films added with $\mathrm{Y}_{2} \mathrm{O}_{3}$ nanoparticulate defects.. Supercond. Sci. Technol. 28, 024002 (2015)

39 Cantoni, C., Gao, Y., Wee, S. H., Specht, E. D., Gazquez, J., Meng, J., Pennycook, S. J. \& Goyal, A. Strain-driven oxygen deficiency in self-assembled, nanostructured, composite oxide films. ACS NANO 5, 4783-4789 (2011).

40 Horide, T., Kitamura, T., Ichinose, A. \& Matsumoto, K. Elastic strain evolution in nanocomposite structure of $\mathrm{YBa}_{2} \mathrm{Cu}_{3} \mathrm{O}_{7}+\mathrm{BaZrO}_{3}$ superconducting films. Jpn. J. Appl. Phys. 53, 083101 (2014).

41 Miura, M., Maiorov, B., Baily, S. A., Haberkorn, N., Willis, J. O., Marken, K., Izumi, T., Shiohara, Y. \& Civale, L. Mixed pinning landscape in nanoparticle-introduced $\mathrm{YGdBa}_{2} \mathrm{Cu}_{3} \mathrm{O}_{y}$ films grown by metal organic deposition. Phys. Rev. B 83, 184519 (2011).

42 Ijaduola, A. O., Thompson, J. R., Feenstra, R., Christen, D. K., Gapud, A. A. \& Song, X. Critical currents of ex situ $\mathrm{YBa}_{2} \mathrm{Cu}_{3} \mathrm{O}_{7-\delta}$ thin films on rolling assisted biaxially textured substrates: thickness, field, and temperature dependencies. Phys. Rev. B 73, 134502 (2006)

43 Solovyov, V. F., Wiesmann, H. J., Wu, L., Li, Q., Cooley, L. D., Suenaga, M., Maiorov, B. \& Civale, L. High critical currents by isotropic magnetic-flux-pinning centres in a 3 micron-thick $\mathrm{YBa}_{2} \mathrm{Cu}_{3} \mathrm{O}_{7}$ superconducting coated conductor. Supercond. Sci. Technol. 20, L20-L23 (2007).

44 Miura, M., Maiorov, B., Kato, T., Shimode, T., Wada, K., Adachi, S. \& Tanabe, K. Strongly enhanced flux pinning in one-step deposition of $\mathrm{BaFe}_{2}\left(\mathrm{As}_{0.66} \mathrm{P}_{0.33}\right)_{2}$ superconductor films with uniformly dispersed $\mathrm{BaZrO}_{3}$ nanoparticles. Nat. Commun. 4, 2499 (2013).

45 Feldmann, D. M., Holesinger, T. G., Maiorov, B., Foltyn, S. R., Coulter, J. Y. \& Apodaca I. Improved flux pinning in $\mathrm{YBa}_{2} \mathrm{Cu}_{3} \mathrm{O}_{y}$ with nanorods of the double perovskite $\mathrm{Ba}_{2} \mathrm{YNbO}_{6}$. Supercond. Sci. Technol. 23, 095004 (2010).

46 Godeke, A. A review of the properties of $\mathrm{Nb}_{3} \mathrm{Sn}$ and their variation with $\mathrm{A} 15$ composition, morphology and strain state. Supercond. Sci. Technol. 19, R68-R80 (2006).

47 Wee, S. H., Zuev, Y. L., Cantoni, C. \& Goyal, A. Engineering nanocolumnar defect configurations for optimized vortex pinning in high temperature superconducting nanocomposite wires. Sci. Rep. 3, 2310 (2013).

48 Selvamanickam, V., Chen, Y., Shi, T., Liu, Y., Khatri, N. D., Liu, J., Yao, Y., Xiong, X., Lei, C., Soloveichik, S., Galstyan, E. \& Majkic, G. High critical currents in heavily doped (Gd,Y)Ba2Cu30x superconductor tapes. Appl. Phys. Lett. 106, 032601 (2015).

49 Civale, L., Marwick, A. D., McElfresh, M. W., Worthington, T. K., Malozemoff, A. P., Holtzberg, F. H., Thompson, J. R. \& Kirk, M. A. Defect independence of the irreversibility line in proton-irradiated Y-Ba-Cu-O crystals. Phys. Rev. Lett. 65, 1164-1167 (1990).

50 Blatter, G., Feilgl'man, M. V., Geshkenbein, V. B., Larkin, A. I. \& Vinokur, V. M. Vortices in high-temperature superconductors. Rev. Mod. Phys. 66 1125-1388 (1994).

51 Matsushita, T., Wada, H., Kiss, T., Inoue, M., Iijima, Y., Kakimoto, K., Saitoh, T. \& Shiohara, Y. Critical current properties in superconducting Y-123 tapes. Phys. C 378-381, 1102-1107 (2002).

52 Koshelev, A. E. \& Kolton, A. B. Theory and simulations on strong pinning of vortex lines by nanoparticles. Phys. Rev. B 84, 104528 (2011).

53 Mishev, V., Zehetmayer, M., Fischer, D. X., Nakajima, M., Eisaki, H. \& Eisterer, M. Interaction of vortices in anisotropic superconductors with isotropic defects. Supercond. Sci. Technol. 28, 102001 (2015).

54 van der Beek, C. J., Konczykowski, M. \& Prozorov, R. Anisotropy of strong pinning in multi-band superconductors. Supercond. Sci. Technol. 25, 084010 (2012).

55 Kramer, E. J. \& Freyhardt, H. C. The proximity effect in flux pinning. J. Appl. Phys. 51 4930-4938 (1980)

56 Long, N. J. Model for the angular dependence of critical currents in technical superconductors. Supercond. Sci. Technol. 21, 025007 (2008). 
57 Wimbush, S. C. \& Long, N. J. The interpretation of the field angle dependence of the critical current in defect-engineered superconductors. New J. Phys. 14, 083017 (2012).

58 Matsui, H., Ogiso, H., Yamasaki, H., Sohma, M., Yamaguchi, I., Kumagai, T. \& Manabe, T. Dimpling in critical current density vs. magnetic field angle in $\mathrm{YBa}_{2} \mathrm{Cu}_{3} \mathrm{O}_{7}$ films irradiated with 3-MeV gold ions. J. Appl. Phys. 114, 233911 (2013).

$59 \mathrm{Li}$, Y., Kang, G. \& Gao, Y. Scaling rules for critical current density in anisotropic biaxial superconductors. Phys. B 491, 70-78 (2016).

60 Malozemoff, A. P. \& Fisher, M. P. A. Universality in the current decay and flux creep of Y-Ba-Cu-O high-temperature superconductors. Phys. Rev. B 42, 6784-6786 (1980)

61 Campbell, A. M. \& Evetts, J. E. Flux vortices and transport currents in type II superconductors. Adv. Phys. 21, 199-428 (1972).

62 Matsushita, T. Flux Pinning in Superconductors, 2nd edn, (Springer: Berlin, 2014).

63 Matsushita, T., Nagamizu, H., Tanabe, K., Kiuchi, M., Otabe, E. S., Tobita, H., Yoshizumi, M., Izumi, T., Shiohara, Y., Yokoe, D., Kato, T. \& Hirayama, T. Improvement of flux pinning performance at high magnetic fields in $\mathrm{GdBa}_{2} \mathrm{Cu}_{3} \mathrm{O}_{y}$ coated conductors with $\mathrm{BHO}$ nano-rods through enhancement of $B_{\mathrm{c} 2}$. Supercond. Sci. Technol. 25 , 125003 (2012).
64 Awaji, S., Namba, M., Watanabe, K., Kai, H., Mukaida, M. \& Okayasu, S. Flux pinning properties of correlated pinning at low temperatures in ErBCO films with inclined columnar defects. J. Appl. Phys. 111, 013914 (2012).

This work is licensed under a Creative Commons Attribution 4.0 International License. The images or other third party material in this article are included in the article's Creative Commons license, unless indicated otherwise in the credit line; if the material is not included under the Creative Commons license, users will need to obtain permission from the license holder to reproduce the material. To view a copy of this license, visit http:// creativecommons.org/licenses/by/4.0/

(C) The Author(s) 2017

Supplementary Information accompanies the paper on the NPG Asia Materials website (http://www.nature.com/am) 